\title{
LOCALLY CONVEX HYPERSURFACES OF NEGATIVELY CURVED SPACES
}

\author{
S. ALEXANDER
}

\begin{abstract}
A well-known theorem due to Hadamard states that if the second fundamental form of a compact immersed hypersurface $M$ of Euclidean space $E^{n}(n>3)$ is positive definite, then $M$ is imbedded as the boundary of a convex body. There have been important generalizations of this theorem concerning hypersurfaces of $E^{n}, H^{n}$, and $S^{n}$, but there seem to be no versions for hypersurfaces of spaces of variable curvature, and no proofs which generalize to these spaces. Our main result is that Hadamard's theorem holds in any complete, simply connected Riemannian manifold of nonpositive sectional curvature.
\end{abstract}

A well-known theorem due to Hadamard states that if the second fundamental form of a compact immersed hypersurface $M$ of Euclidean space $E^{n}$ $(n \geqslant 3)$ is positive definite, then $M$ is imbedded as the boundary of a convex body. There have been important generalizations of this theorem concerning hypersurfaces of $E^{n}, H^{n}$, and $S^{n}$ (for example, see Remark 4). However, there seem to be no versions of the theorem for hypersurfaces of spaces of variable curvature, and no proofs which generalize to these spaces. Theorem 1 states that Hadamard's theorem holds in any complete, simply connected Riemannian manifold $N$ of nonpositive sectional curvature; in this paper, $N$ always denotes such a space. We refer to [2] for notation and background material.

Proposition 1. Let $i: M \rightarrow N$ be a hypersurface immersion of a compact, connected, orientable manifold $M$ of dimension $m \geqslant 2$, and $Z$ be a continuous unit normal. If the sectional curvatures of $N$ satisfy $K_{N}<-k<0$ and $Z$ may be chosen so that the eigenvalues of the second fundamental form $S_{Z}$ satisfy $\lambda \geqslant-\sqrt{k}$, then $M$ is diffeomorphic to the sphere $S^{m}$.

Proof. Note that here $S_{Z}$ is defined by $S_{Z}(X, Y)=\left\langle\nabla_{X} Z, Y\right\rangle$, for vector fields $X, Y$ on $M$. Since $M$ is compact, $i(M)$ lies in some metric ball $B$. Since $K_{N} \leqslant 0, \exp _{y}$ is a diffeomorphism onto $N$, for any $y \in N$, and metric balls are convex. Therefore for any $x \in M$, the geodesic ray $j_{x}$ in $N$ with initial direction $Z(x)$ strikes $\partial B$ once and transversely, say at the point $p(x)$. If $M$ has no focal points on $j_{x}$, then the map $p: M \rightarrow \partial B$ is a diffeomorphism, since $\partial B$ is diffeomorphic to $S^{m}$. Thus the proposition follows from an extension of

Received by the editors June 14, 1976 and, in revised form, November 17, 1976.

AMS (MOS) subject classifications (1970). Primary 53C40, 53C20.

( American Mathematical Society 1977 
the Rauch comparison theorem due to Warner [11], whereby since there are no focal points in the case where $N$ has constant curvature $K_{N}=-k$ and $M$ is a horosphere with $\lambda=-\sqrt{k}$, then there are none in our case.

REMARK 1. If $K_{N} \leqslant 0$ and $\lambda \geqslant 0, M$ is imbedded. If $K_{N} \leqslant-k$, one might conjecture existence of a bound $b(k),-\sqrt{k} \leqslant b(k)<0$, such that $M$ is imbedded if $\lambda \geqslant b(k)$. To see that no such $b(k)$ exists, let $N=H^{n}$, regarded as the Euclidean open unit disk $D^{n}$ carrying the Poincare metric of constant curvature -1 . For any $\varepsilon>0$ and any ball $B$ about the origin in $D^{n}$, there exists an immersion $i: S^{n-1} \rightarrow B$ which is not an imbedding and whose Euclidean second fundamental form with respect to a continuous unit normal satisfies $\lambda \geqslant-\varepsilon$. Indeed, let $i_{0}: S^{n-1} \rightarrow B$ bound a convex body enclosing a flat $(n-1)$-disk $D$; it is easy to see that if $d\left(i_{0}(x), D\right) \leqslant \delta$ for all $x \in S^{n-1}$, where $\delta$ is sufficiently small and $d$ is Euclidean distance, then $i_{0}$ may be deformed so as to acquire self-intersections and still satisfy the inequality $\lambda>-\varepsilon$ with respect to the outer unit normal. Now let the radius of $B$ be $\varepsilon / 2$. It may be verified that for any immersion in $B$ with Euclidean form satisfying $\lambda \geqslant-\varepsilon$, the hyperbolic form with respect to the corresponding hyperbolic unit normal also satisfies $\lambda \geqslant-\varepsilon$.

THEOREM 1. Let $i: M \rightarrow N$ be a hypersurface immersion of a compact, connected, orientable manifold $M$ of dimension $m \geqslant 2$, and $Z$ be a continuous unit normal. If $Z$ may be chosen so that $S_{Z}$ is positive semidefinite, then $M$ is imbedded in $N$ as the boundary of a convex body.

Proof. By the preceding proof, we have the diffeomorphism $p: M \rightarrow \partial B$. Define $j: M \times(-\varepsilon, \infty) \rightarrow N$ by $j(x, c)=\exp c Z(x)$, and let $j_{x}$ be the geodesic ray obtained by fixing $x$, and $j_{c}$ be the mapping of $M$ into $N$ obtained by fixing $c$. By a simple verification, $j$ is an immersion if $\varepsilon$ is sufficiently small, and $j_{c}$ has positive semidefinite second fundamental form if $c \geqslant 0$. (Indeed, if $Z$ is the vector field along $j$ given by $Z(x, c)=j_{x}^{\prime}(c)$, then any field $X$ on $M$ determines a field $X$ along $j$ orthogonal to $Z$, by $X(x, c)=j_{c *} X(x)$. Along $j_{x}, X$ is Jacobi, so $\langle X, X\rangle$ is convex and $2\left\langle S_{Z} X, X\right\rangle=Z \cdot\langle X, X\rangle$ is nondecreasing.) By a theorem of Bishop [1], semidefiniteness implies that at each $x \in M, j_{c}$ is locally convex away from $Z(x, c)$. That is, there exist $\delta>0$ and neighborhoods $U$ of $x$ in $M$ and $V$ of 0 in $i_{*} T_{x} M$ such that $\exp V$ does not intersect $j(U \times(c-\delta, c))$.

Suppose $i$ is not an imbedding, so that there exist distinct $x, y \in M$ for which $j_{0}(x)=i(x)=i(y)$. Define $c \geqslant 0$ by

$$
c=\max \left\{d: j_{d}(x)=i(y) \text { for some } x, y \in M\right\} \text {. }
$$

We may choose points $x, y$ satisfying: (i) $j_{c}(x)=i(y)$; (ii) $x, y$ do not have connected neighborhoods $U, V$ respectively such that $j_{c}(U)$ and $i(V)$ are the same topologically imbedded hypersurface. Indeed, if $x$ and $y$ are distinct points satisfying (i) but having such neighborhoods $U$ and $V$, then injectivity of $p$ implies that $j_{c}(V)=i(U)$ (note that $U$ and $V$ are interchanged), so $i$ is totally geodesic on $U$ and $V$. Thus if $\alpha:[0, a] \rightarrow M$ is any path from $x$ to a 
point at which $i$ is not totally geodesic, there exists a path $\beta:[0, b] \rightarrow M$ from $y$ such that $j_{c} \circ \alpha(t)=i \circ \beta(t), 0 \leqslant t \leqslant b \leqslant a$, and (i) and (ii) are satisfied by the new choice $x=\alpha(b), y=\beta(b)$.

By maximality of $c, j(M \times(c, \infty))$ does not intersect $i(M)$. Therefore, by (i), the immersions $j_{c}$ and $i$ are tangential at $x$ and $y$ respectively, and $Z(x, c)= \pm Z(y, 0)$; furthermore, by (ii) and local convexity, $Z(x, c) \neq-$ $Z(y, 0)$. But then $p(x)=p(y)$, a contradiction. We conclude that $i$ is an imbedding. Therefore $N-i(M)$ consists of two open components with boundary $i(M)$, one bounded and one unbounded. $Z$ points away from one component, say $A$. From local convexity of the boundary of $A$, it follows that $A$ is convex (the argument is the same for $N$ as for $E^{n}$, and is given in [8], as the Schmidt Theorem). Since the complement of a proper convex subset of $N$ is unbounded, $i(M)$ is the boundary of a bounded convex open set.

REMARK 2. It is interesting to inquire whether the usual Morse-theoretic approach to such problems in $E^{n}$ might be generalized to immersions in $N$. A "Gauss map", which agrees with the usual one if $N=E^{n}$, could be defined from the unit normal bundle of $M$ to the $(n-1)$-sphere $N_{\infty}$. Here $N_{\infty}$ consists of asymptote classes of geodesic rays [6]; a unit normal $Z_{x}$ would map to the class of the ray with initial tangent $Z_{x}$. Analogues of the Euclidean height functions are available in the Busemann functions, whose level hypersurfaces are horospheres. These functions do not seem helpful in dealing with semidefiniteness of the second fundamental form of $M$, but considering them leads to the following observation.

Proposition 2. Let $i: M \rightarrow N$ be an isometric hypersurface immersion of a complete, connected Riemannian manifold $M$ of dimension $m$. If the sectional curvatures of $N$ satisfy $K_{N} \leqslant-k \leqslant 0$ and the eigenvalues of the second fundamental form of $i$ satisfy $|\lambda| \leqslant \sqrt{k}$, then $M$ is diffeomorphic to $E^{m}$ and imbedded.

Proof. For any $z \in N$, consider the function $f_{z}(x)=d(i(x), z)$ on $M$, where $d$ is distance in $N$. Let $\alpha$ be an $M$-geodesic parametrized by arclength. Then any critical point $c$ of $f_{z} \circ \alpha$ is a strict relative minimum point. (If $N=H^{n}$, this claim follows from a lemma of Ferus [7].) Otherwise, $i \circ \alpha$ would be tangent at $c$ to a metric sphere of positive radius about $z$, and would intersect the corresponding closed metric ball arbitrarily close to $c$. If $S_{Y}$ is the second fundamental form of the sphere, where $Y$ is the outer unit normal at $i \circ \alpha(c)$, and if $T=(i \circ \alpha)^{\prime}$, then it follows that $\left.\left|\left\langle\nabla_{T} T, Y\right\rangle\right|\right\rangle S_{Y}(T, T)$. This is impossible, since $S_{Y}(T, T)>\sqrt{k}$ by the Rauch comparison theorem, whereas $\left\|\nabla_{T} T\right\| \leqslant \sqrt{k}$ by hypothesis and the fact that $\alpha$ is an $M$-geodesic.

If $i \circ \alpha(a)=i \circ \alpha(b)=z$, then $a=b$, so geodesics in $M$ are imbedded under $i$, and $M$ is imbedded also. By hypothesis, $K_{M} \leqslant 0$, so $M$ has no conjugate points. Thus if the exponential map of $M$ at $x \in M$ is not a diffeomorphism, there exists a nontrivial $M$-geodesic $\alpha$ joining $x$ to itself, and we have just shown this is impossible. Note that sublevel sets $f_{z}^{-1}[0, c]$ are 
totally convex in $M$ (contain every geodesic between any two points), so $i$ is globally supported by any tangent metric sphere in $N$. In particular, it follows that $i$ is globally supported by both tangent horospheres at every point $i(x)$, $x \in M$.

REMARK 3. Theorem 1 fails completely if the curvature restriction on $N$ is removed. For example, for any compact immersed hypersurface $M$ of $E^{n}$ having sufficiently nice self-intersections, one may construct on a tubular neighborhood a Riemannian metric with respect to which $M$ is totally geodesic. Combining this metric with the standard metric on $E^{n}$ via a Urysohn function which vanishes off of a compact set, one obtains a complete metric on $E^{n}$ with respect to which $M$ is totally geodesic.

Furthermore, an imbedded sphere $M$ with positive semidefinite second fundamental form in a complete, simply connected Riemannian manifold need not bound a convex set (a set containing exactly one minimal geodesic between any two points); Karcher considers this problem in relation to the cut locus of $M$ in [9].

REMARK 4. Hadamard's theorem was extended to compact oriented hypersurfaces of $E^{n}$ having (unsigned) semidefinite second fundamental form by Chern and Lashof [5], and Sacksteder [10] (see proof in [3]). Do Carmo and Warner have proved that this version also holds in $H^{n}$ and $S^{n}$ [4]; their method uses geodesic mappings to recover the Euclidean situation. It would be interesting to discover whether this version holds in nonpositively curved spaces $N$, and also to find versions which hold under other curvature hypotheses on the receiving space.

Note ADDED IN PROOF. Professor do Carmo informs me that Mr. Ivan Tribuzy has been considering similar problems. Among his results is the special case of Theorem 1 in which $M$ is assumed to have positive sectional curvature.

\section{REFERENCES}

1. R. L. Bishop, Infinitesimal convexity implies local convexity, Indiana Univ. Math. J. 24 (1974/75), 169-172. MR 50 \#3154.

2. R. L. Bishop and R. J. Crittenden, Geometry of manifolds, Academic Press, New York, 1964. MR 29 \#6401.

3. M. P. do Carmo and E. Lima, Isometric immersions with semi-definite second quadratic forms, Arch. Math. (Basel) 20 (1969), 173-175. MR 39 \#6214.

4. M. P. do Carmo and F. W. Warner, Rigidity and convexity of hypersurfaces in spheres, J. Differential Geometry 4(1970), 133-144. MR 42 \# 1014.

5. S. S. Chern and R. K. Lashof, On the total curvature of immersed manifolds, Amer. J. Math. 79 (1957), 306-318; II, Mich. Math. J. 5 (1958), 5-12. MR 18, 927; 20 \# 4301.

6. P. Eberlein and B. O’Neill, Visibility manifolds, Pacific J. Math. 46 (1973), 45-109. MR 49 \#1421.

7. D. Ferus, On isometric immersions between hyperbolic spaces, Math. Ann. 205 (1973), 193-200. MR 49 \# 1438.

8. J. van Heijenoort, On locally convex manifolds, Comm. Pure Appl. Math. 5 (1952), 223-242. MR 14, 577. 
9. H. Karcher, Schnittort and konvexe Mengen in vollständigen Riemannschen Mannigfaltigkeiten, Math. Ann. 177 (1968), 105-121. MR 37 \#2131.

10. R. Sacksteder, On hypersurfaces with no negative sectional curoatures, Amer. J. Math. 82 (1960), 609-630. MR 22 \# 7087.

11. F. W. Warner, Extension of the Rauch comparison theorem to submanifolds, Trans. Amer. Math. Soc. 122 (1966), 341-356. MR 34 \#759.

Departmant of Mathematics, University of Illinois at Urbana-Champaign, Urbana, ILLINOIS 61801 